\title{
Management of Penetrating Skull Base Injury: A Single Institutional Experience and Review of the Literature
}

\author{
Danfeng Zhang, Jigang Chen, Kaiwei Han, Mingkun Yu, and Lijun Hou \\ Department of Neurosurgery, Shanghai Institute of Neurosurgery, Shanghai Changzheng Hospital, \\ 415 Fengyang Road, Shanghai 200003, China \\ Correspondence should be addressed to Lijun Hou; lijunhoucz@126.com
}

Received 7 May 2017; Accepted 18 June 2017; Published 30 July 2017

Academic Editor: Hideo Inaba

Copyright (C) 2017 Danfeng Zhang et al. This is an open access article distributed under the Creative Commons Attribution License, which permits unrestricted use, distribution, and reproduction in any medium, provided the original work is properly cited.

Background. Penetrating skull base injury (PSBI) is uncommon among head injuries, presenting unique diagnostic and therapeutic challenges. Although many cases of PSBIs have been reported, comprehensive understanding of its initial diagnosis, management, and outcome is still unavailable. Materials and Methods. A retrospective review was performed for patients treated in neurosurgical department of Changzheng Hospital for PSBIs. Presurgical three-dimensional (3D) Slicer-assisted reconstructions were conducted for each patient. Then we reviewed previous literature about all the published cases of PSBIs worldwide and discussed their common features. Results. A total of 5 patients suffering PSBIs were identified. Penetrating points as well as the surrounding neurovascular structures were clearly visualized, assisting in the presurgical planning of optimal surgical approach and avoiding unexpected vascular injury. Four patients underwent craniotomy with foreign bodies removed successfully and 1 patient received conservative treatment. All of them presented good outcomes after proper management. Conclusion. Careful physical examination and radiological evaluation are essential before operation, and angiography is recommended for those with suspected vascular injuries. 3D modeling with 3D Slicer is practicable and reliable, facilitating the diagnosis and presurgical planning. Treatment decision should be made upon the comprehensive evaluation of patient's clinicoradiological features and characteristics of foreign bodies.

\section{Introduction}

Penetrating injuries of the skull base caused by foreign bodies are relatively uncommon, representing about $0.4 \%$ of head injuries [1, 2]. Various foreign bodies have been reported in penetrating skull base injuries (PSBIs), including wood, bamboo, metallic fragments, and toothbrushes. PSBIs could present unique diagnostic and therapeutic challenges. Patients may be initially asymptomatic but subject to serious events for several days, months, or even years after the injuries [3]. It is not difficult to detect most of intracranial foreign bodies by head computed tomography (CT) scan. However, the relationships between foreign bodies and its surrounding structures are hard to decide, which is the prerequisite for the management of these injuries $[4,5]$.

Treatment for PSBIs includes the surgical retraction of foreign bodies, prevention of infection, management of vascular injuries, reconstruction of skull base, and so forth [3, $5-7]$. Due to the low incidence, there is no sufficient literature about the diagnosis and management of these patients. The aim of our study is to present patients of PSBIs treated in our department and review related literature in order to highlight the proper management of PSBIs and improve prognosis in the long run.

\section{Materials and Methods}

A retrospective review was conducted for patients who were treated in our hospital between January 2010 and September 2016 for PSBIs. This study was performed in accordance with the Declaration of Helsinki (1964) and approved by the investigational review board of Changzheng Hospital. Informed consent was available for each patient. Presurgical head CT scans were performed for all patients. Moreover, digital subtraction angiography (DSA) was conducted on suspicion of vascular injuries and magnetic resonance imaging (MRI) was performed in case of nonmetallic objects. Data concerning patients' demographics, mechanisms of 
injury, medical managements, complications, and prognosis were collected by two authors (D. F. Zhang and J. G. Chen).

In order to visualize the location of foreign body and its relationship with surrounding structures, three-dimensional (3D) Slicer-assisted reconstructions were conducted by a professional neuroradiologist (K. W. Han) according to presurgical imaging data. During the reconstruction, all the Digital Imaging and Communications in Medicine (DICOM) images were imported into 3D Slicer (3D Slicer 4.0 4.4; Surgical Planning Laboratory, Harvard University, USA). Segmentation of skull, foreign body, and cerebral artery was first performed with built-in modules in Slicer. Individual models of each structure were created, which could be rotated and viewed from any perspective (Figure $1(\mathrm{~g})$; Figures 2(g)-2(i); Figure 3(g)).

\section{Results}

A total of 5 patients with PSBIs were identified. There were 4 males and 1 female aged $29-75$ years. They were all victims of tumble or work-related accident. Four of them underwent surgical retrieval of foreign bodies and 1 patient received conservative treatment. Penetrating points as well as the surrounding neurovascular structures were clearly visualized in 3D models, assisting in the presurgical planning of optimal surgical approach and avoiding unexpected vascular injury. Details regarding patients' demographics, locations of foreign bodies, and treatment were listed in Table 1.

3.1. Representative Case 1. This 75-year-old female was admitted to her local hospital with complaints of headache and dizziness for 3 days. She was conscious and neurologically intact with a slightly elevated body temperature. Head CT scan revealed a low density $4 \mathrm{~cm}$ long foreign body extending from the left orbit to superior orbital fissure and posteriorly to the left temporal lobe (Figures 1(a)-1(c)). Brain tissue surrounding the foreign body was swollen and signs of abscess were indicated (Figures $1(\mathrm{~d})-1(\mathrm{f})$ ). The patient recalled that while she was walking in a bamboo garden, she tripped and fell forward, striking her left forehead on a bamboo stick. She did not feel any discomfort except the pain on her left upper eyelid. Symptoms of headache and fever emerged 5 days later and she was taken to the hospital by her family 8 days after the injury. Anti-infective therapies were given before she was transferred to our hospital. Careful physical examination revealed a slight skin scar on her left upper eyelid. Head DSA was performed later with no signs of vascular injuries although the bamboo stick was adjacent to the left middle cerebral artery (MCA) in the 3D reconstruction model (Figure $1(\mathrm{~g})$ ). Anti-infectious treatment was administrated for 12 days to control the brain abscess before surgery. During operation, an orbitozygomatic approach was adopted and the pterional craniotomy was first performed. Then superior orbital fissure was revealed after removing the great wing of sphenoid. We explored the abscess in the temporal lobe. After yellowish pus in the abscess was removed, the distal end of the bamboo stick was then visualized. We opened the dura and orbital fascia along the bamboo stick to expose its full length (Figure $1(\mathrm{~h})$ ). The stick was removed completely under direct visualization (Figure 1(i)) and dura was sutured in a water tight fashion. The postoperative course was uncomplicated. Broad-spectrum antibiotics were given until she was discharged free of symptoms 10 days after operation.

3.2. Representative Case 2. This 32-year-old man was hit by the fragments of a burst grinding wheel on his left cheek during working hours. He was taken to the local hospital complaining of headache and kept neurologically intact on physical examination. Head CT scan suggested a short piece of metal fragment locating right between the left maxillary and rami mandibulae and a long piece sticking in the left temporal lobe. Both pieces presented to be highly dense on the CT scan (Figures 2(a)-2(c)). The patient was then transferred to our department for further treatment 5 days later. DSA suggested no obvious vascular injury (Figures 2(d) and 2(e)), while axial CT scan revealed close relationship between the bone fragment and branch of MCA (Figure 2(f)). The exact location of foreign body could be visualized clearly on the $3 \mathrm{D}$ reconstruction model (Figures $2(\mathrm{~g})-2(\mathrm{i})$ ). Conservative management was given to control the infection and surgery was performed 15 days after the injury. During operation, short piece of the foreign body was first removed through an intraoral incision. Then a temporozygomatic approach was performed to remove the long piece, which was visualized at the infratemporal fossa after the zygomatic arch was detached and retracted downwardly with temporal muscle. Several pieces of bone fragment were found around the foreign body. After the lateral portion of infratemporal fossa was drilled off, the object was removed in a retrograde fashion. During this process, yellowish pus was drained into infratemporal fossa through the intracranial trajectory of foreign body. This trajectory was not explored and defect of dura was sealed with muscle flap (Figures 3(a)-3(e)). Postoperative skull radiography showed complete removal of the foreign body (Figure 3(f)). A small bone fragment was left in place due to its close relationship with branch of MCA, which was found to be stenotic during follow-up (Figure 3(g)). The patient was discharged 1 week after operation with sporadic focal epilepsy which was controlled well with carbamazepine, and he recovered well without abscess formation at threemonth follow-up (Figures 3(h) and 3(i)).

\section{Discussion}

4.1. Literature Review. Clinicoradiological features of PSBIs in previous literatures were reviewed and summarized in Table 2 [2, 3, 8-24]. Most of the subjects were males (66\%, 21/32) with an average age of 24 years old. $41 \%$ (13/32) of the patients were children under 10 years of age. As for the mechanism of injury, children injuries were all caused by tumbling or falling, while the injury of 4 adults resulted from suicidal or homicidal attempt, which was absent in our case series. The most common foreign body was metallic $(12 / 32)$, followed by wooden (9/32) and plastic (9/32). One patient was injured by a wild deer's antler and another one was attacked by bear paws, both of which had no retained foreign bodies. As a weak area of skull, the orbit was the most 


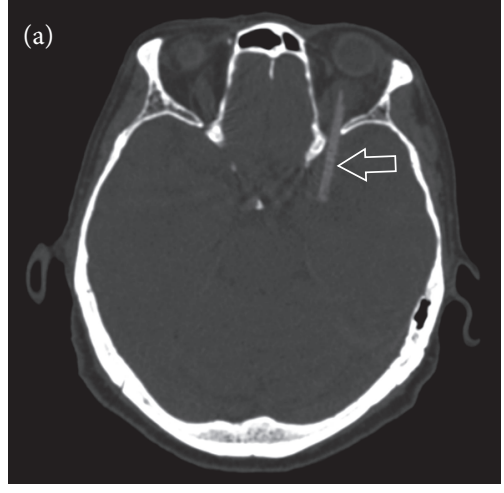

(a)

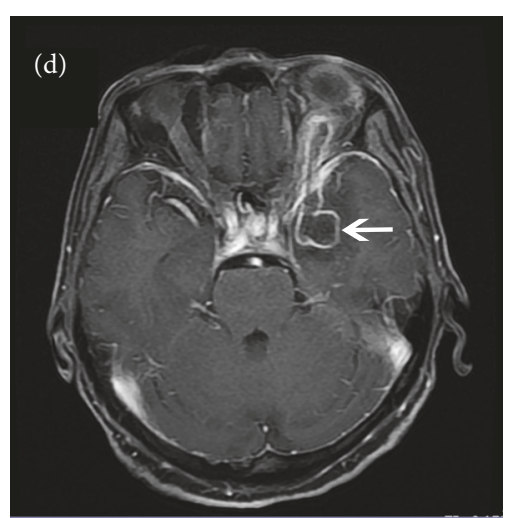

(d)

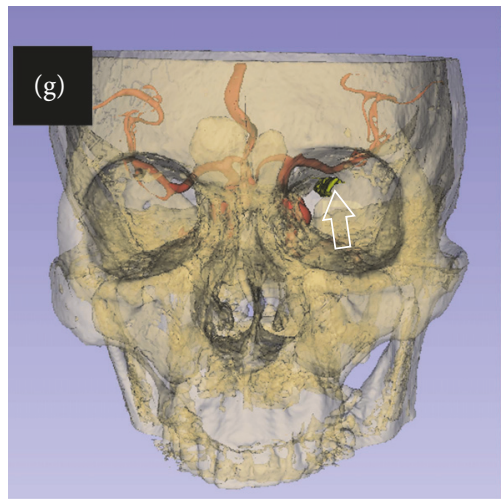

(g)

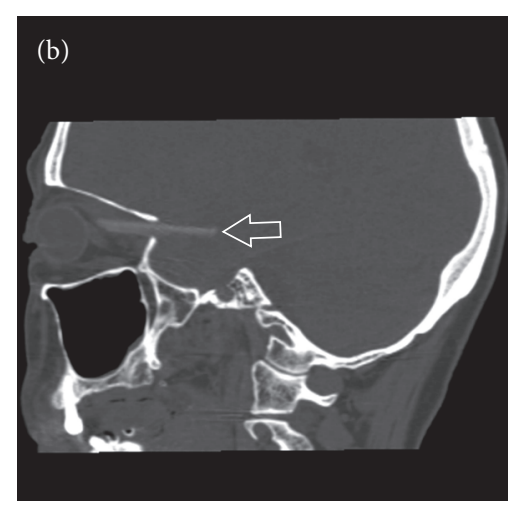

(b)

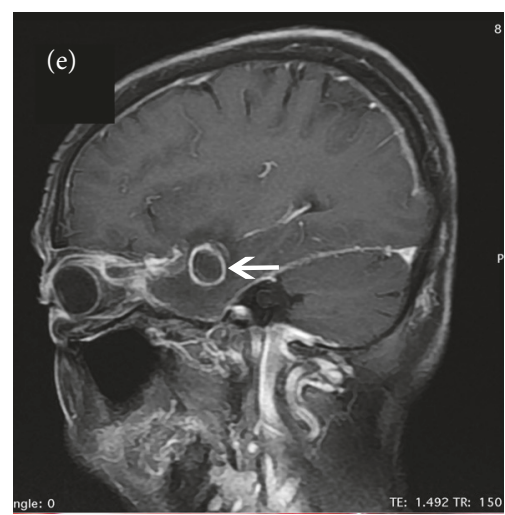

(e)

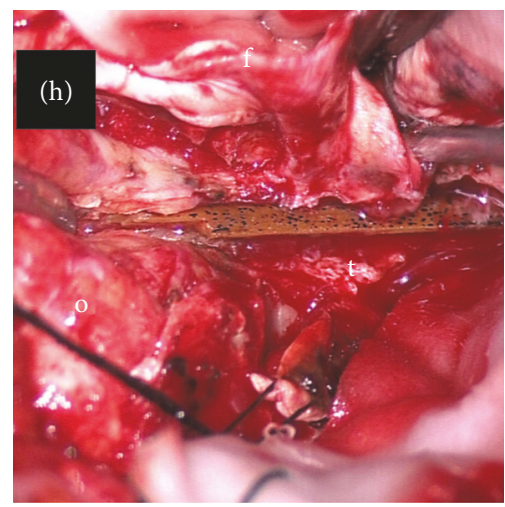

(h)

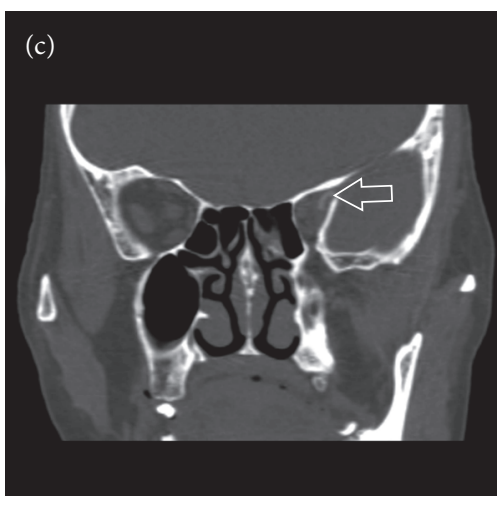

(c)

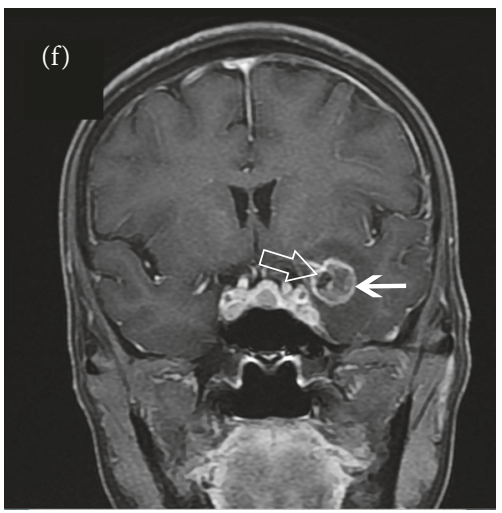

(f)

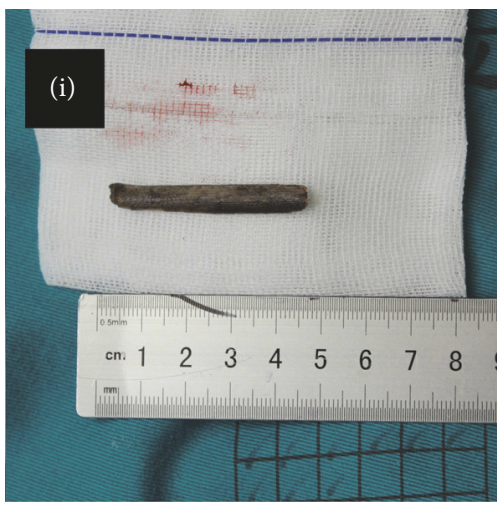

(i)

FIGURE 1: Head CT scan demonstrated a bamboo stick (hollow arrow, $\hookleftarrow$ ) penetrating into the temporal lobe via superior orbital fissure. The bamboo stick presented as high density on the CT scan (a, b, c). Contrast enhanced MRI revealed an abscess (simple arrow, $\leftarrow$ ) around the bamboo stick in temporal lobe (d, e, f). 3D reconstruction of the skull, cerebral artery, and bamboo stick (hollow arrow, $\bullet$ ) was performed by $3 \mathrm{D}$ Slicer software to visualize the relationship among these structures ( $\mathrm{g}$ ). Intraoperative photography (h) displayed the bamboo stick in original place (o, orbital side; $\mathrm{f}$, frontal side; t, temporal side). Photography showed the removed bamboo stick (i). 3D, three-dimensional; CT, computed tomography; MRI, magnetic resonance imaging.

common penetrating point, followed by oral or nasal cavity and maxillofacial region.

4.2. Diagnosis of PSBIs. After careful physical examination, proper radiological examination on the basis of patients' condition is necessary. Although the importance of head CT scan in the management of PSBIs has been emphasized in previous literatures, several instructions should be noticed. Firstly, the density of foreign bodies on CT scan varies according to their types. For example, metal presents as high density, while wood or plastics are of isodensity or low density and difficult to identify. Secondly, the density of some foreign bodies would change over time. For example, bamboo is of low density on initial CT scan, but it would be of high density 


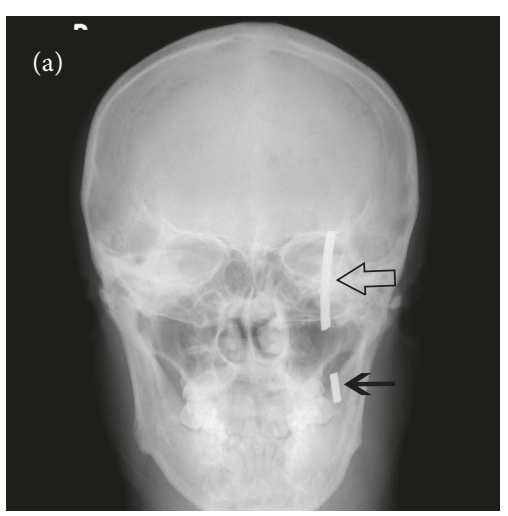

(a)

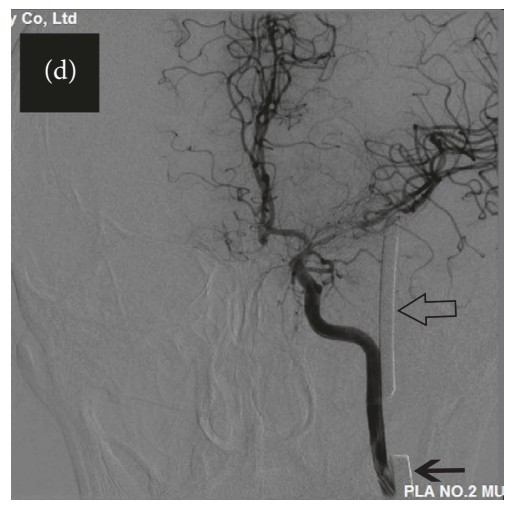

(d)

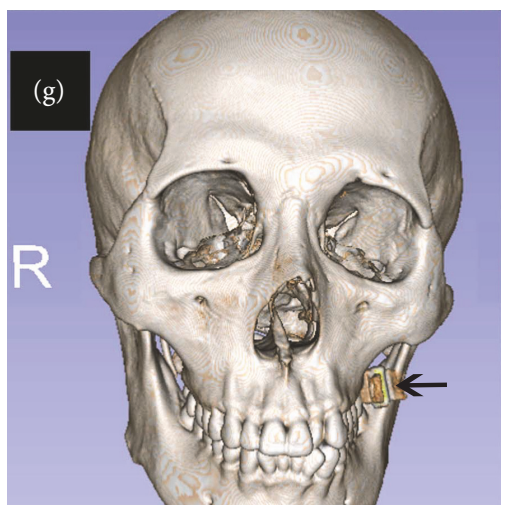

(g)

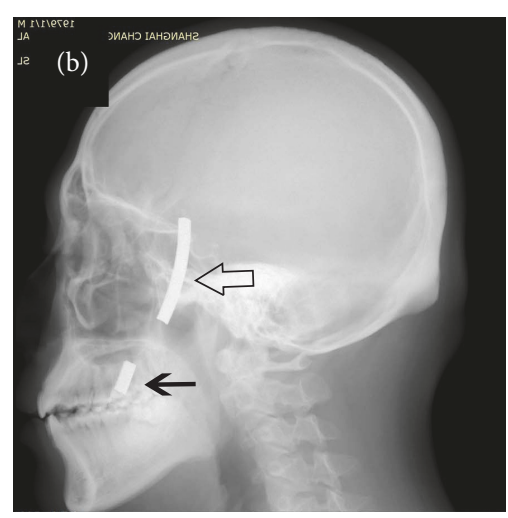

(b)

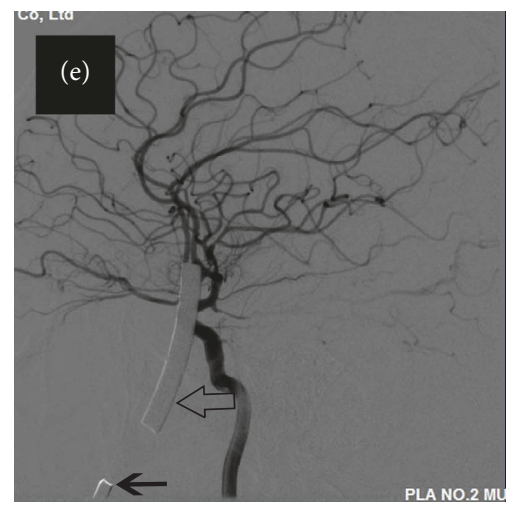

(e)

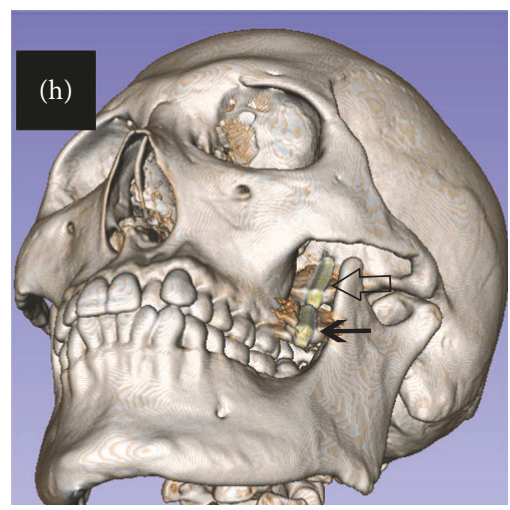

(h)

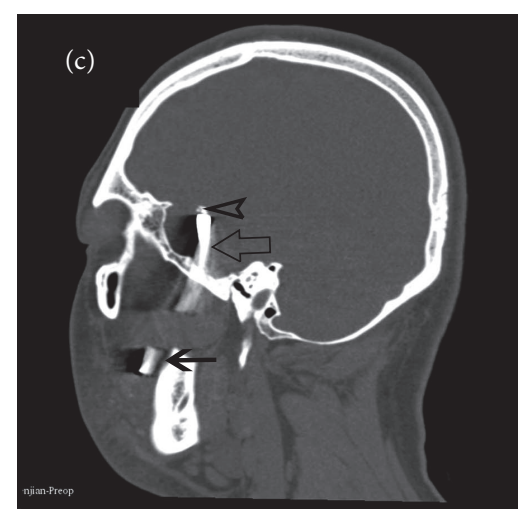

(c)

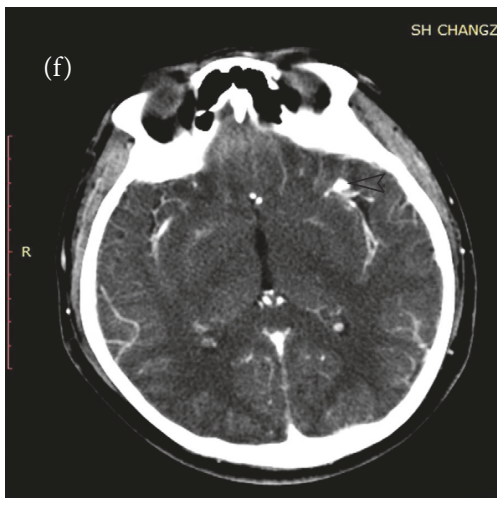

(f)

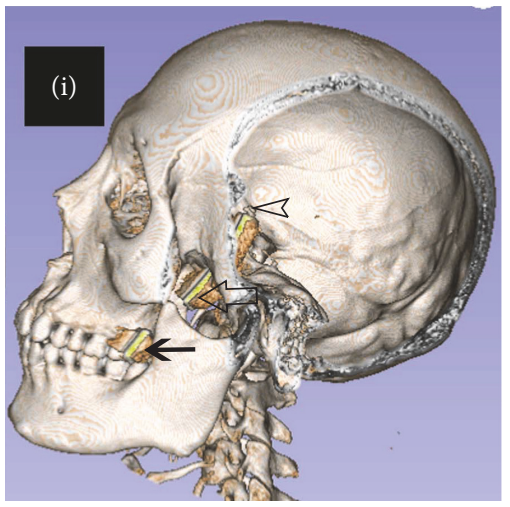

(i)

FIGURE 2: Preoperative image of foreign body (burst outer rim of a grinding wheel) in the face and middle skull base. Anterior-posterior skull radiography (a) and lateral skull radiography (b) demonstrated the short piece (simple arrow, $\leftarrow$ ) in the face and long piece (hollow arrow, $\diamond)$ penetrating into the middle skull base. Sagittal reconstruction of CT (c) showed the long piece penetrating into the middle cranial fossa through infratemporal fossa. A small piece of bone fragment (hollow arrowhead, $>$ ) was noticed above the long piece. The bone fragment arose from the hit of the long piece on the middle skull base. DSA ( $\mathrm{d} \& \mathrm{e}$ ) proved the integrity of MCA. Axial CT revealed close relationship between the bone fragment (hollow arrow head, $>$ ) and branch of MCA (f). 3D reconstruction (g, h, i) with 3D Slicer software displayed spatial correlation of two pieces of foreign body with the face and skull base. 3D, three-dimensional; CT, computed tomography; DSA, digital subtraction angiography; MCA, middle cerebral artery.

later, which frequently leads to misdiagnosis (Case 1) [25]. Therefore, MRI is a useful supplement to CT in the detection of nonmetallic foreign bodies.

Angiography such as the CT angiography, MR angiography, or DSA is highly recommended for patients in suspicion of artery injuries or traumatic aneurysms $[7,26,27]$. As demonstrated in Case 2, foreign bodies may not cause immediate vascular injury sometimes but lead to cerebral vasospasm or stenosis in the long run, highlighting the importance of angiography in the setting of PSBIs. 


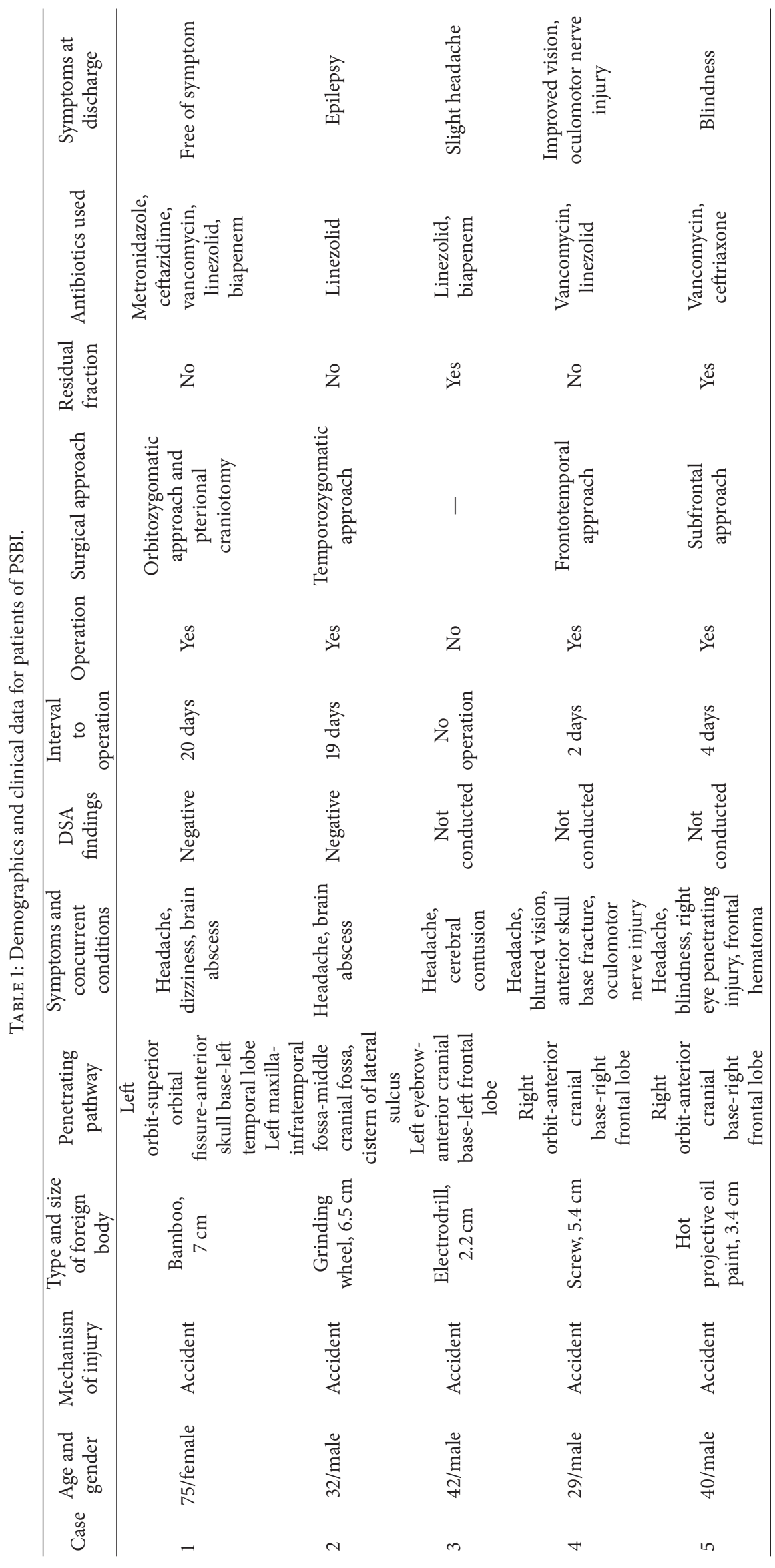




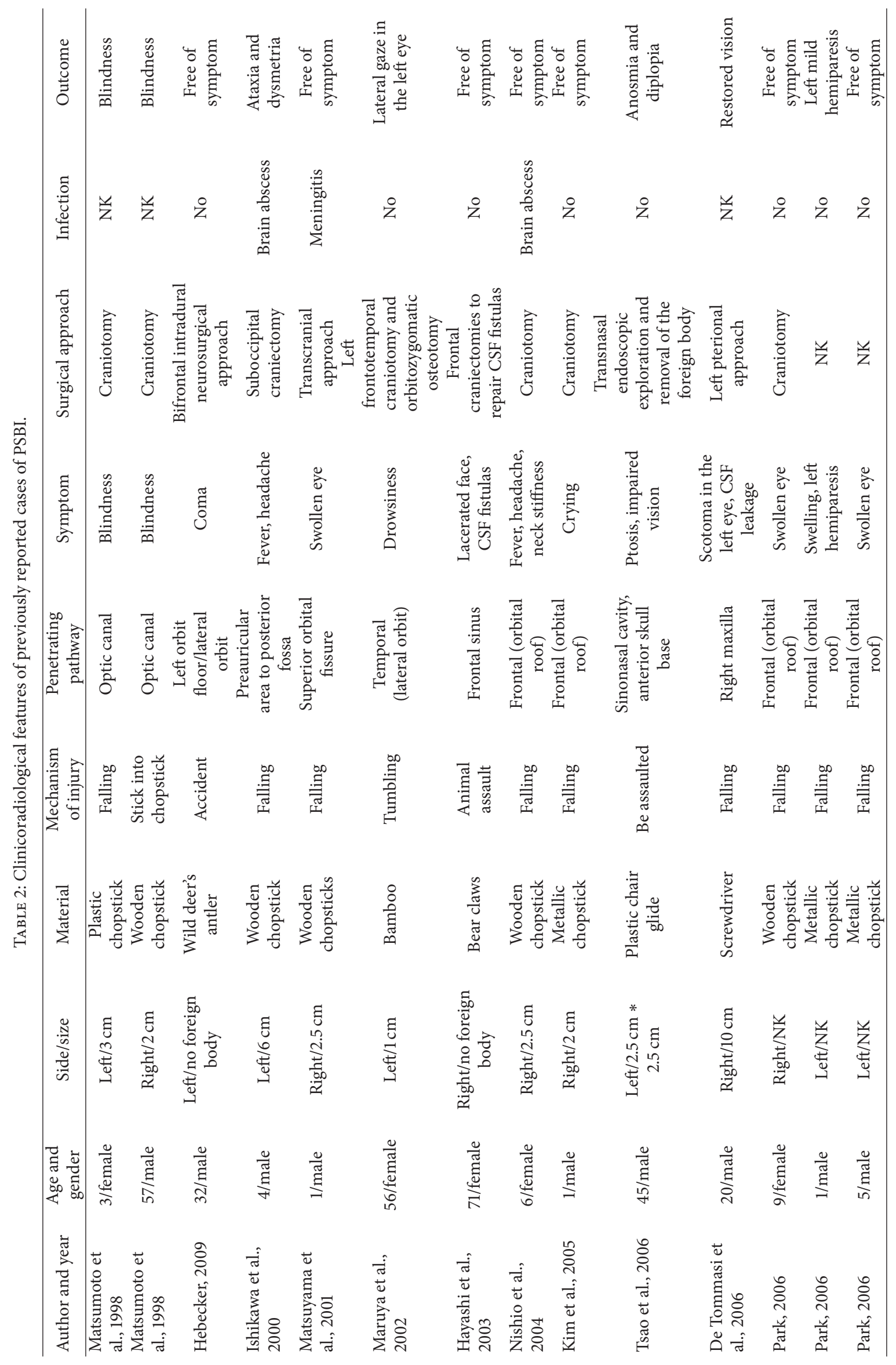




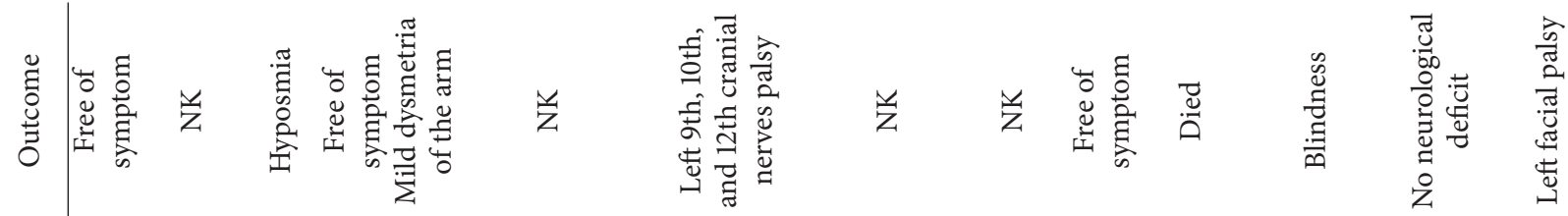

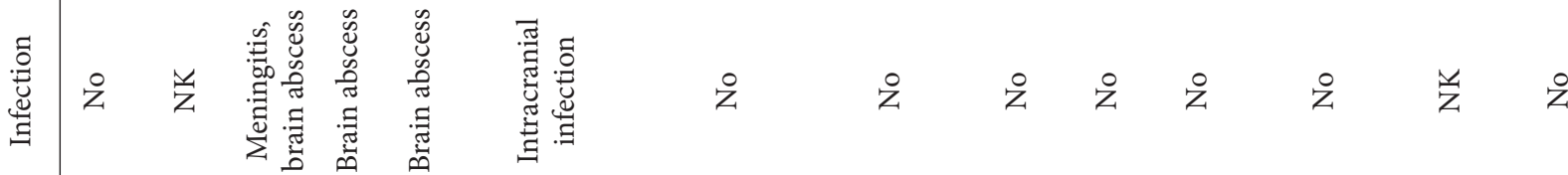

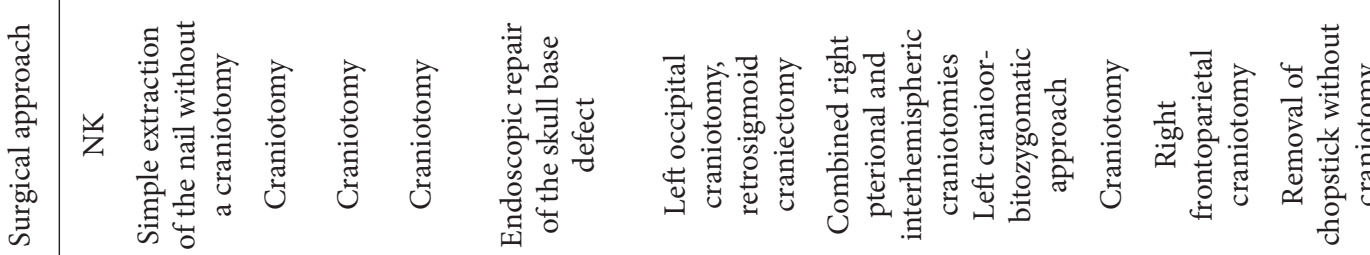

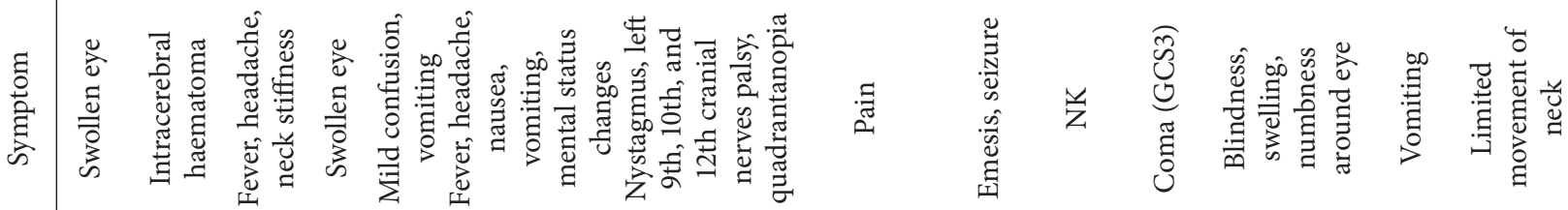

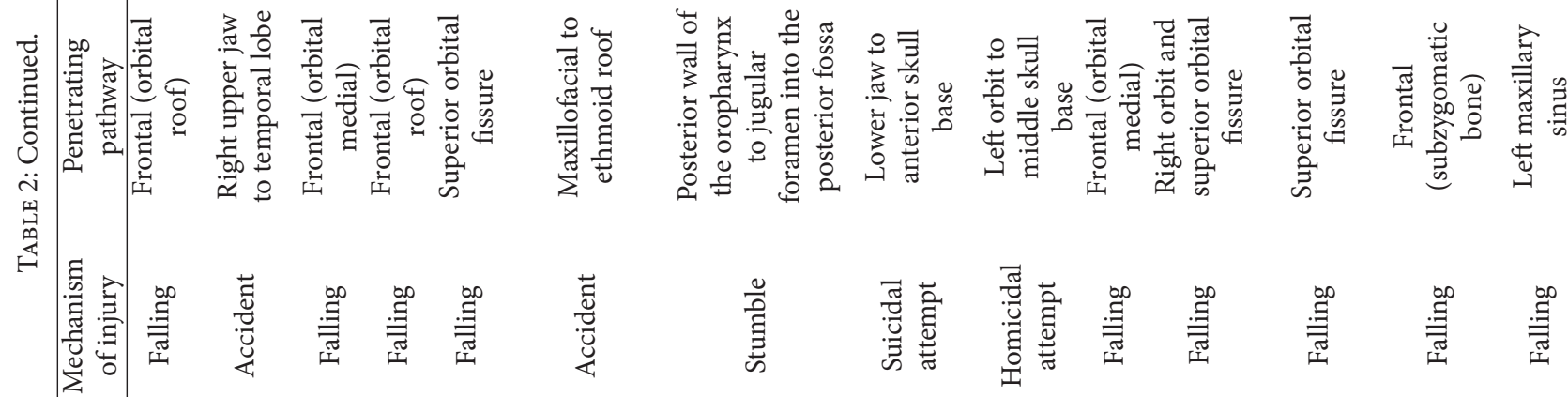

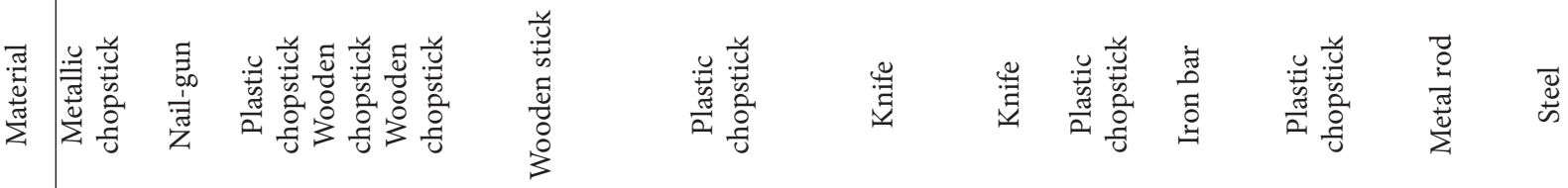

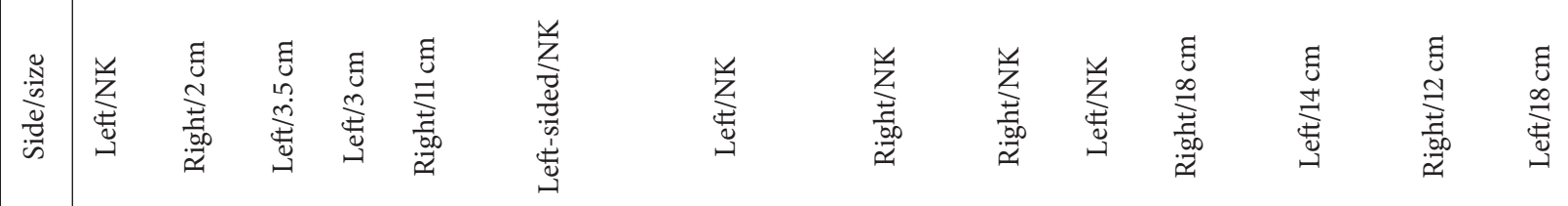

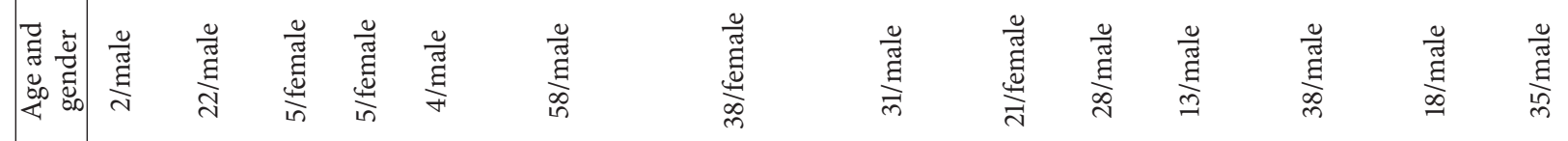

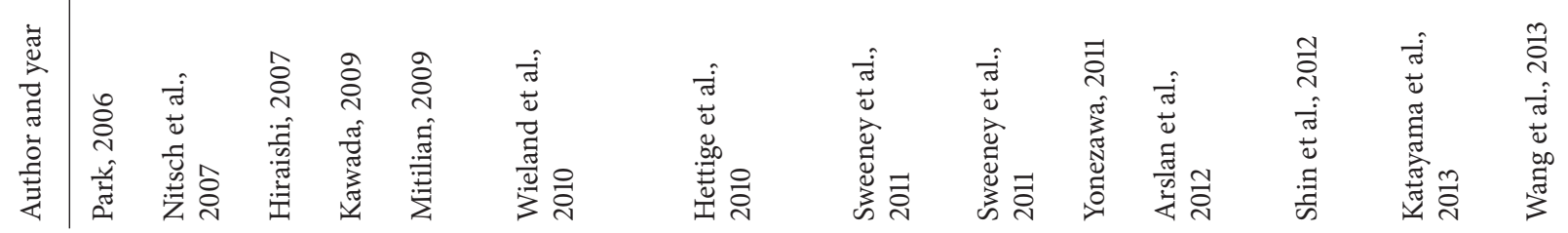




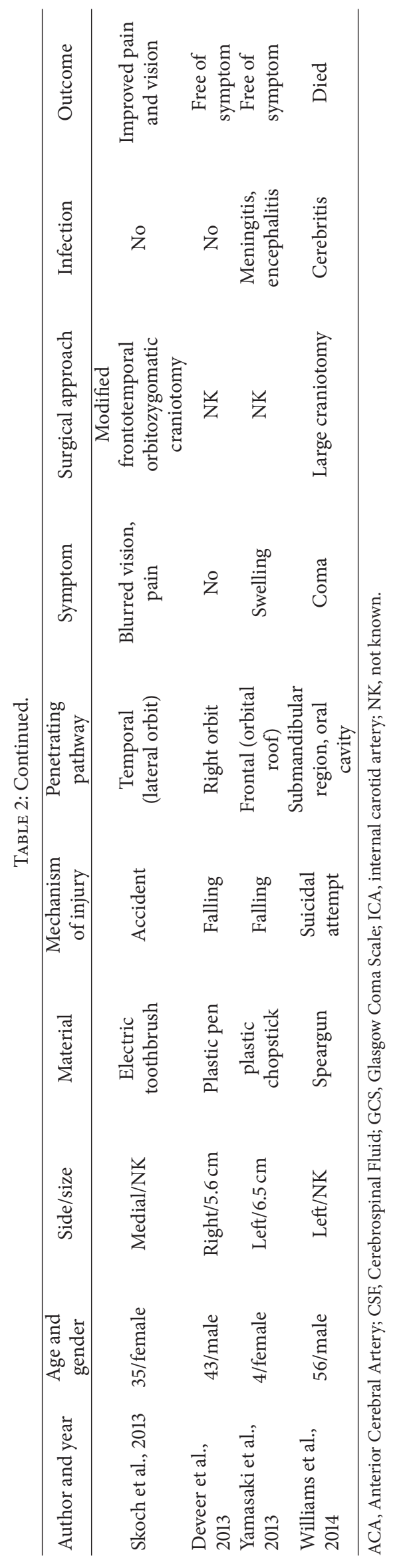




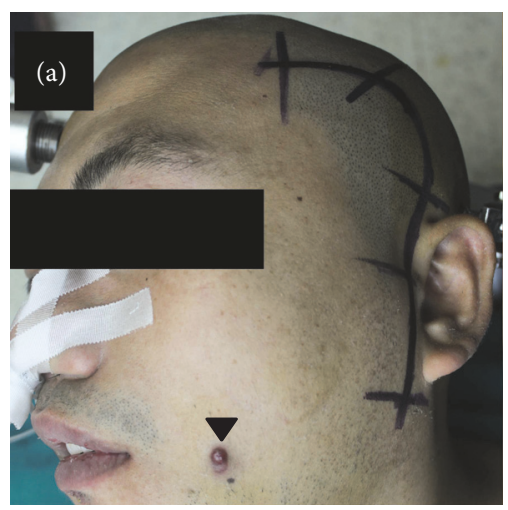

(a)

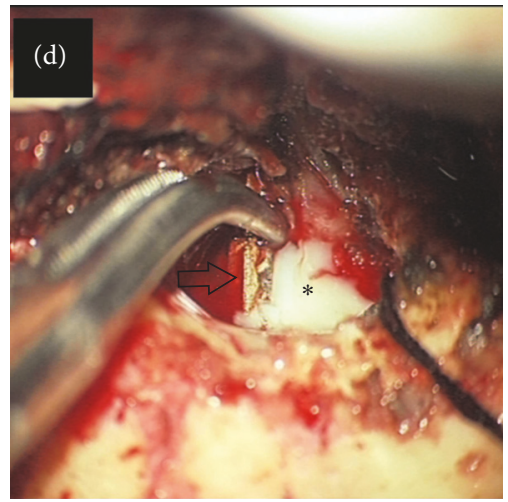

(d)

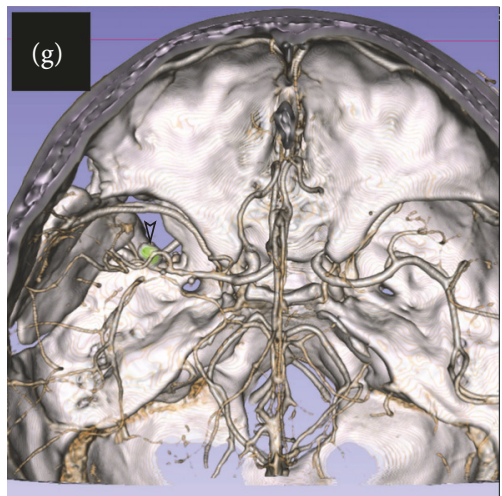

(g)

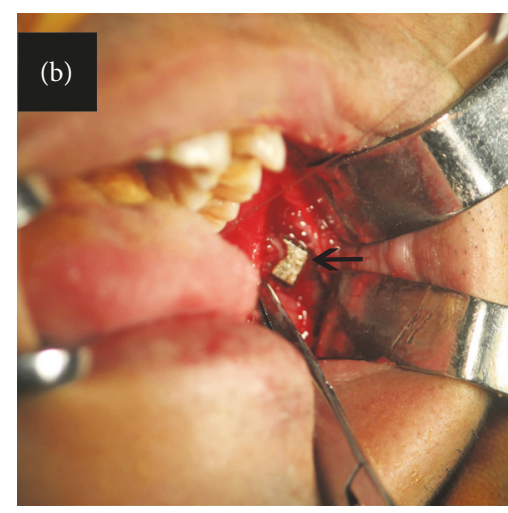

(b)

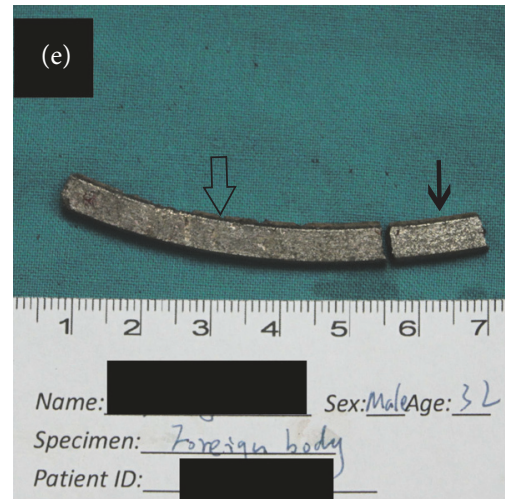

(e)

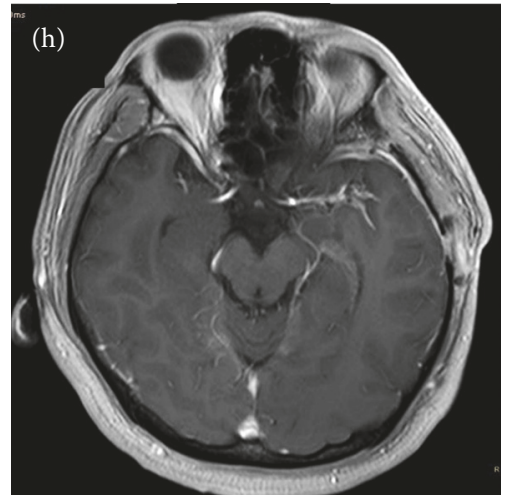

(h)

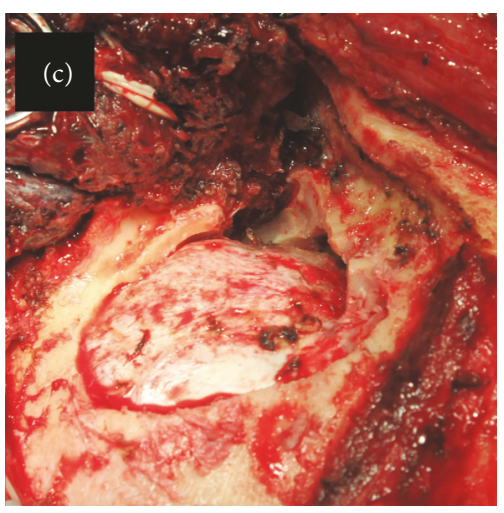

(c)

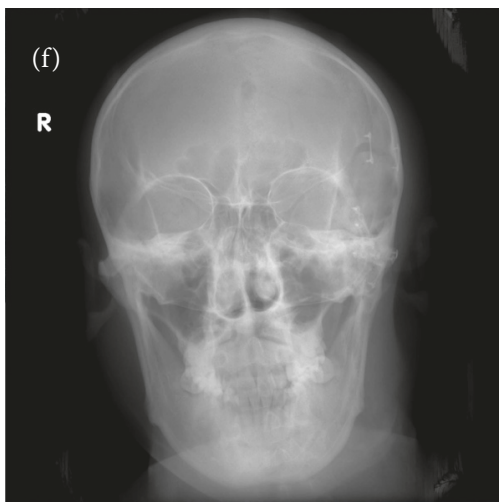

(f)

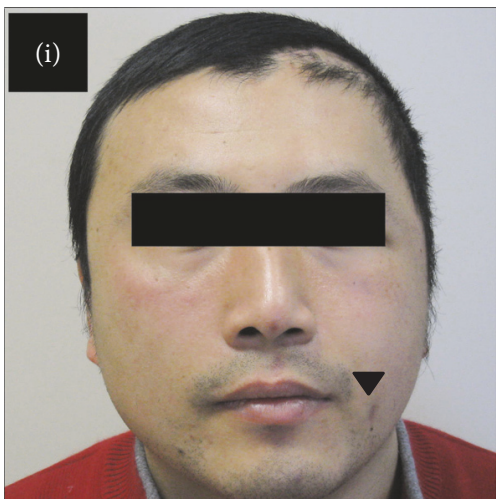

(i)

FIGURE 3: Photography showed the entry point (black triangle, $\mathbf{\nabla}$ ) of foreign body on face and incision for a frontotemporoorbitozygomatic approach (a). Short piece of foreign body (simple arrow, $\leftarrow$ ) in the face was exposed and removed by a maxillofacial surgeon (b). The infratemporal fossa was opened to expose the long piece of foreign body (c), and it (hollow arrow, $\hookleftarrow$ ) was removed in a retrograde fashion. During the removing process, yellowish pus (asterisk, $*$ ) was drained into the infratemporal fossa $(\mathrm{d})$. Photography displayed short piece (simple arrow, $\leftarrow$ ) and long piece (hollow arrow, $\bullet$ ) of the foreign body (e). Postoperative skull radiography suggested complete removal of the foreign body (f). The small bone fragment (hollow arrow head, $>$ ) was left in place due to its close relationship with branch of MCA (g). Three-month follow-up MRI revealed no abscess formation (h). The patient recovered uneventfully (i). MCA, middle cerebral artery; MRI, magnetic resonance imaging.

3D modeling has been frequently used in the presurgical planning of PSBIs in previous studies $[8,11,15-17,19,23$, $24,28]$. 3D models can be viewed in 360 degrees, rotated, and studied from any perspective [29-31], which facilitate the diagnosis and operation to some extent [30, 31]. 3D Slicer is a free, open source software that can be used for segmentation and 3D modeling with high accuracy and reliability $[30,32]$. It has been used in the management of various diseases including intracranial aneurysms, trigeminal neuralgia, and intracerebral hematomas $[29,30,33]$. PSBIs in our case series were reconstructed using 3D Slicer, in which penetrating points as well as the surrounding neurovascular structures 
were clearly visualized. It contributed to the presurgical planning of optimal surgical approach and avoiding unexpected vascular injury. Our findings confirmed the feasibility and reliability of 3D Slicer in the modeling of foreign objects and adjacent neurovascular structures. Moreover, the segmentation and modeling procedure using 3D Slicer allowed higher quality of visualization, better view of objects and richer information than workstation reconstruction (Figures $1(\mathrm{~g})$, 2(g)-2(i), and 3(g)) [30]. To our knowledge, this is the first study to visualize PSBIs with $3 \mathrm{D}$ Slicer, granting preoperative surgical plan for proper approaches.

4.3. Treatment of PSBIs. Owing to the low incidence of PSBIs, prospective or controlled study is difficult to conduct on the limited cases. Temporary management of PBSIs constantly depends on the experience of different institutions $[7,15]$. Despite the availability of some complicated guidelines for penetrating brain injury, they were mostly based on the data of scattered cases without systematic summary for PSBIs [3, 8-10]. Thus, we discussed the treatment of PSBIs based on related cases we treated and previously reported.

Operation is the major strategy for the treatment of PSBIs. Indications for surgery are retained objects, CSF leakage, fracture displacement, intracranial hemorrhage, and vascular injury [34-38]. The purpose of surgery is to remove foreign objects, decompress brain tissue, and reconstruct skull base. Generally, operation is suggested in 12 hours after PSBIs [39, 40]. However, delayed operation is not recommended until full physical and radiological examination are performed since premature surgery might lead to fatal results. Artery injury is one of the common concurrent conditions that should be identified before surgery, the presence of which will be a disaster for emergent operation [16].

In PSBIs with vascular injuries, protection of injured arteries through preoperative endovascular occlusion or intraoperative artery control would be helpful in the removal of foreign bodies [41]. In contrast, in PSBIs without vascular injuries, foreign bodies could be removed directly [7]. For some typical cases, the metal foreign bodies or bone fragments are adjacent to important structures and difficult to extract but cause no obvious symptoms. These foreign bodies could be retained in the brain (Case 2).

Surgical approach for skull base injury should be individualized according to the penetrating trajectory, location of foreign body, and accompanying vascular and brain injuries. In current study, different modifications of frontotemporoorbitozygomatic approach were adopted to manage the anterior and middle skull base injuries. By removing the superior and lateral bony orbit, we could deal with most of foreign bodies penetrating from the orbit into frontal lobe. The removal of zygomatic arch enables inferior displacement of temporalis muscle, allowing exploration of undersurface of temporal lobe. Generally, principles for skull base surgery can also be applied to PSBIs. Proximal vascular control should be first guaranteed to prevent intraoperative hemorrhage. And direct visualization of foreign object should be achieved before its removal. Usually, it is necessary to drill away the bone of skull base to expose the foreign bodies. Thorough debridement along the exposed trajectory as well as careful reconstruction of the skull base is of great significance to prevent postoperative infection and CSF leakage. However, aggressive debridement for deep seated debris should be avoided, which may be associated with increased disability and mortality [20].

Infection is the main complication of PSBIs with a reported overall rate of $64-70 \%$ and mortality rate of $14-57 \%$ [42-44]. Organic foreign body like wood or bamboo is not only the carrier, but also the best medium for infection such as brain abscess, meningitis, and cerebritis [36, 45]. In this way, organic foreign bodies should be totally retrieved, while some other deep seated foreign bodies such as small metal or bone fragments could be retained since total extraction would cause more damage. In the absence of sufficient data and definitive guidelines, the type, timing, and duration of antibiotic use remains uncertain, especially when the result of CSF culture is negative. In recent publications, prophylactic use of broad-spectrum antibiotic was suggested within 7-14 days after the injury [16, 39], while others indicated that antibiotic therapy should be administrated according to the findings of CSF culture [46]. We recommended prophylactic use of antibiotics and proper adjustment according to CSF culture results, especially for wooden objects.

\section{Conclusions}

PSBI is a rare disease with various injury mechanisms and complicated traumatic conditions. Lots of difficulties regarding the diagnosis and management of PSBIs remain to be solved. Based on our experience and review of previous studies, we suggest full physical examination and radiological evaluation before surgery. Preoperative 3D modeling with 3D Slicer could help visualize penetrating pathway and surrounding neurovascular structures in detail, granting free view from any angle and selection of optimal approach. However, caution is needed in interpreting our findings because of the limited cases. Further large scale prospective studies are required to identify the effect of preoperative $3 \mathrm{D}$ reconstruction on the prognosis of PSBIs, as well as the guideline for the management of PSBIs.

\section{Conflicts of Interest}

The authors declare that there are no conflicts of interest regarding the publication of this article.

\section{Authors' Contributions}

Danfeng Zhang performed data collection, data analysis, and writing of the article. Jigang Chen collaborated in literature search and study design; Kaiwei Han collaborated in figure generation and study design; Mingkun Yu collaborated in study design, data analysis, and editing of the article; Lijun Hou collaborated in literature research, figure generation, and editing of the article. 


\section{References}

[1] T. A. Gennarelli, H. R. Champion, W. J. Sacco, W. S. Copes, and W. M. Alves, "Mortality of patients with head injury and extracranial injury treated in trauma centers," Journal of Trauma, vol. 29, no. 9, pp. 1193-1201, 1989.

[2] Y. Hayashi, H. Fujisawa, Y. Tohma, J. Yamashita, and H. Inaba, "Penetrating head injury caused by bear claws: case report," Journal of Trauma - Injury, Infection and Critical Care, vol. 55, no. 6, pp. 1178-1180, 2003.

[3] J. Maruya et al., "Brain abscess following transorbital penetrating injury due to bamboo fragments-case report," Neurol Med Chir (Tokyo), vol. 42, no. 3, pp. 143-146, 2002.

[4] M. S. Walid, J. C. Yelverton, and J. S. Robinson Jr., "Penetrating orbital trauma with internal carotid injury," Southern Medical Journal, vol. 102, no. 1, pp. 116-117, 2009.

[5] A. Agrawal, A. Pratap, C. S. Agrawal, A. Kumar, and S. Rupakheti, "Transorbital orbitocranial penetrating injury due to bicycle brake handle in a child," Pediatric Neurosurgery, vol. 43, no. 6, pp. 498-500, 2007.

[6] F. H. Chowdhury, M. R. Haque, Z. Hossain, N. K. Chowdhury, S. M. Alam, and M. H. Sarker, "Nonmissile Penetrating Injury to the Head: Experience with 17 Cases," World Neurosurgery, vol. 94, pp. 529-543, 2016.

[7] M. Schreckinger, D. Orringer, B. G. Thompson, F. La Marca, and O. Sagher, "Transorbital penetrating injury: Case series, review of the literature, and proposed management algorithm: Report of 4 cases," Journal of Neurosurgery, vol. 114, no. 1, pp. 53-61, 2011.

[8] M. Deveer, F. Imamoglu, C. Imamoglu, and S. Okten, "An incidental case of asymptomatic intracranial foreign body on CT," BMJ case reports, vol. 2013, 2013.

[9] S. Hettige, K. Kok, P. Epaliyanage, and N. W. M. Thomas, "Chopstick injury penetrating the skull base: A case report," Skull Base, vol. 20, no. 3, pp. 219-222, 2010.

[10] A. De Tommasi, P. Cascardi, C. De Tommasi, S. Luzzi, and P. Ciappetta, "Emergency surgery in a severe penetrating skull base injury by a screwdriver: Case report and literature review," World Journal of Emergency Surgery, vol. 1, no. 1, article 36, 2006.

[11] J. Skoch, T. L. Ansay, and G. M. Lemole, "Injury to the temporal lobe via medial transorbital entry of a toothbrush," Journal of Neurological Surgery Reports, vol. 74, no. 1, pp. 23-28, 2013.

[12] E. Ishikawa, K. Meguro, K. Yanaka et al., "Intracerebellar penetrating injury and abscess due to a wooden foreign body-case report," Neurologia Medico-Chirurgica, vol. 40, no. 9, pp. 458$462,2000$.

[13] S. Matsumoto, K. Hasuo, A. Mizushima et al., "Intracranial penetrating injuries via the optic canal," AJNR. American Journal of Neuroradiology, vol. 19, no. 6, pp. 1163-1165, 1998.

[14] A. M. Wieland, W. T. Curry, M. L. Durand, and E. H. Holbrook, "Management of a long-standing organic intracranial foreign body," Skull Base, vol. 20, no. 6, pp. 487-490, 2010.

[15] J. M. Sweeney, J. J. Lebovitz, J. L. Eller, J. R. Coppens, R. D. Bucholz, and S. I. Abdulrauf, "Management of nonmissile penetrating brain injuries: a description of three cases and review of the literature," Skull Base, vol. 1, no. 1, pp. 39-46, 2011.

[16] J. R. Williams, D. M. Aghion, C. E. Doberstein, R. G. Cosgrove, and W. F. Asaad, "Penetrating brain injury after suicide attempt with speargun: case study and review of literature," Frontiers in Neurology, vol. 5, article 113, 2014.

[17] A. Nitsch, R. Verheggen, and H.-A. Merten, "Penetrating pneumatic nail-gun injury to skull base," British Journal of Oral and Maxillofacial Surgery, vol. 45, no. 8, p. 692, 2007.
[18] Y. Wang, L. Pan, and H. Xu, “The surgical treatment of reinforced steel bar injury penetrating the skull base and maxillamandibular area," Journal of Craniofacial Surgery, vol. 25, no. 6, pp. e521-e523, 2014.

[19] K. Katayama, N. Shimamura, Y. Ogasawara, M. Naraoka, and H. Ohkuma, "Translucent three-dimensional CT is useful in considering the treatment strategy for the penetrating skull base injury with a metal rod: Case report," Neurologia MedicoChirurgica, vol. 53, no. 9, pp. 613-615, 2013.

[20] M. Arslan, M. Eseoglu, B. O. Güdü, and I. Demir, "Transorbital orbitocranial penetrating injury caused by a metal bar," Journal of Neurosciences in Rural Practice, vol. 3, no. 2, pp. 178-181, 2012.

[21] T. Matsuyama et al., "Transorbital penetrating injury by a chopstick-case report," Neurol Med Chir (Tokyo), vol. 41, no. 7, pp. 345-348, 2001.

[22] Y.-H. Tsao, C.-H. Kao, H.-W. Wang, S.-C. Chin, and K. S. Moe, "Transorbital penetrating injury of paranasal sinuses and anterior skull base by a plastic chair glide: Management options of a foreign body in multiple anatomic compartments," Otolaryngology - Head and Neck Surgery, vol. 134, no. 1, pp. 177179, 2006.

[23] F. Yamasaki, H. Ohge, R. Tsumura et al., "Transorbital penetrating intracranial injury by a chopstick: a case report and review of the literature," No Shinkei Geka, vol. 41, no. 11, pp. 1001-1009, 2013.

[24] S. Kim, J. Y. Lee, J. S. Song, and J. Oh, “Transorbital-intracranial injury by a chopstick: Three-dimensional computed tomography," Acta Ophthalmologica Scandinavica, vol. 83, no. 5, pp. 609610, 2005.

[25] H. Imokawa, T. Tazawa, N. Sugiura, D. Oyake, and K. Yosino, "Penetrating neck injuries involving wooden foreign bodies: the role of MRI and the misinterpretation of CT images," Auris Nasus Larynx, vol. 30, pp. S145-S147, 2003.

[26] A. Carothers, "Orbitofacial Wounds and Cerebral Artery Injuries Caused by Umbrella Tips," JAMA: The Journal of the American Medical Association, vol. 239, no. 12, pp. 1151-1152, 1978.

[27] R. Eidsness, D. J. Coupal, M. E. B. Kelly, and S. Hattingh, "Traumatic orbital injury," Journal of Trauma-Injury, Infection and Critical Care, vol. 62, no. 5, pp. 1286-1287, 2007.

[28] T.-H. Shin, J.-H. Kim, K.-W. Kwak, and S.-H. Kim, "Transorbital penetrating intracranial injury by a chopstick," Journal of Korean Neurosurgical Society, vol. 52, no. 4, pp. 414-416, 2012.

[29] X. Xu, X. Chen, J. Zhang et al., "Comparison of the tada formula with software slicer: Precise and low-cost method for volume assessment of intracerebral hematoma," Stroke, vol. 45, no. 11, pp. 3433-3435, 2014.

[30] K.-W. Han, D.-F. Zhang, J.-G. Chen, and L.-J. Hou, "Presurgical visualization of the neurovascular relationship in trigeminal neuralgia with 3D modeling using free Slicer software," Acta Neurochirurgica, vol. 158, no. 11, pp. 2195-2201, 2016.

[31] B. You, Y. Cheng, J. Zhang et al., "Application of contrastenhanced T1-weighted MRI-based 3D reconstruction of the dural tail sign in meningioma resection," Journal of Neurosurgery, vol. 125, no. 1, pp. 46-52, 2016.

[32] A. Fedorov, R. Beichel, J. Kalpathy-Cramer et al., "3D slicer as an image computing platform for the quantitative imaging network," Magnetic Resonance Imaging, vol. 30, no. 9, pp. 13231341, 2012.

[33] A. Can, A. Mouminah, A. L. Ho, and R. Du, "Effect of vascular anatomy on the formation of basilar tip aneurysms," Neurosurgery, vol. 76, no. 1, pp. 62-66, 2015. 
[34] J. Fezza and R. Wesley, "The Importance of CT Scans in Planning the Removal of Orbital-Frontal Lobe Foreign Bodies," Ophthalmic Plastic \& Reconstructive Surgery, vol. 15, no. 5, pp. 366-368, 1999.

[35] K. A. Greene, C. A. Dickman, K. A. Smith, E. J. Kinder, and J. M. Zabramski, "Self-inflicted orbital and intracranial injury with a retained foreign body, associated with psychotic depression: Case report and review," Surgical Neurology, vol. 40, no. 6, pp. 499-503, 1993.

[36] S. A. Sadiq and G. Thurairajan, "A case of transorbital intracranial damage underlying a seemingly innocuous injury," Injury, vol. 26, no. 4, pp. 279-280, 1995.

[37] K. A. Szabo, S. H. Cheshier, M. Y. S. Kalani, J. W. Kim, and R. Guzman, "Supraorbital approach for repair of open anterior skull base fracture: case report," Journal of Neurosurgery: Pediatrics, vol. 2, no. 6, pp. 420-423, 2008.

[38] T. Yamashita, T. Mikami, T. Baba et al., "Transorbital intracranial penetrating injury from impaling on an earpick," Journal of Neuro-Ophthalmology, vol. 27, no. 1, pp. 48-49, 2007.

[39] S. F. Kazim, M. S. Shamim, M. Z. Tahir, S. A. Enam, and S. Waheed, "Management of penetrating brain injury," Journal of Emergencies, Trauma and Shock, vol. 4, no. 3, pp. 395-402, 2011.

[40] T. S. Helling, W. Kendall McNabney, C. Keith Whittaker, C. C. Schultz, and M. Watkins, "The role of early surgical intervention in civilian gunshot wounds to the head," Journal of Trauma-Injury, Infection and Critical Care, vol. 32, no. 3, pp. 398-400, 1992.

[41] E. J. Cunningham, B. Albani, T. J. Masaryk et al., “Temporary balloon occlusion of the cavernous carotid artery for removal of an orbital and intracranial foreign body: technical case report," Neurosurgery, vol. 55, no. 5, p. 1225, 2004.

[42] Y. Nishio, N. Hayashi, H. Hamada, Y. Hirashima, and S. Endo, "A case of delayed brain abscess due to a retained intracranial wooden foreign body: a case report and review of the last 20 years," Acta Neurochir (Wien), vol. 146, no. 8, pp. 847-850, 2004.

[43] C. F. Miller, J. S. Brodkey, and B. J. Colombi, "The danger of intracranial wood," Surg Neurol, vol. 7, no. 2, pp. 95-103, 1977.

[44] Q. Chunhua and W. Qun, "A late-onset seizure due to a retained intracranial foreign body-pencil lead: a case report and review," Journal of Craniofacial Surgery, vol. 25, no. 2, pp. e109-e110, 2014.

[45] I. F. Dunn, D. H. Kim, P. A. Rubin, R. Blinder, J. Gates, and A. J. Golby, "Orbitocranial wooden foreign body: a pre-, intra-, and postoperative chronicle: case report," Neurosurgery, vol. 65, no. 2, pp. E383-E384, 2009.

[46] R. Gutiérrez-González, G. R. Boto, M. Rivero-Garvía, Á. PérezZamarrón, and G. Gómez, "Penetrating brain injury by drill bit," Clinical Neurology and Neurosurgery, vol. 110, no. 2, pp. 207-210, 2008. 

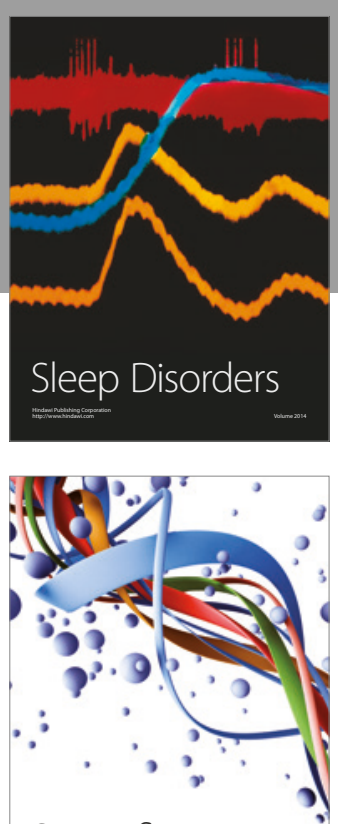

Scientifica
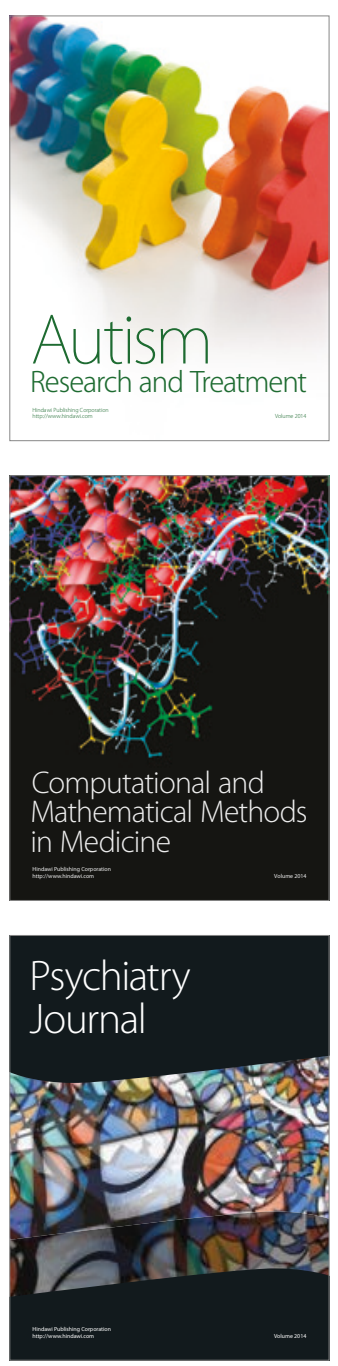
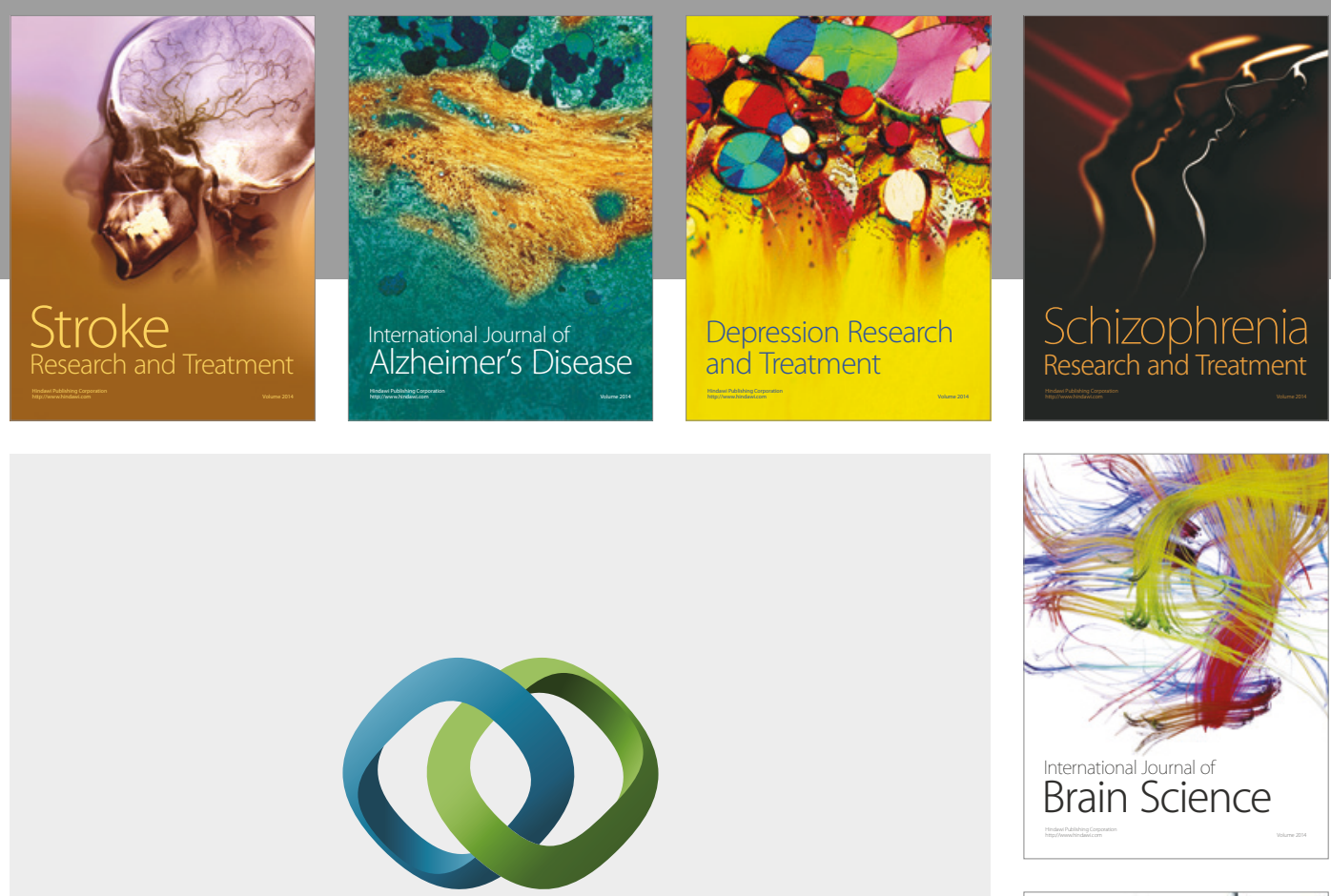

\section{Hindawi}

Submit your manuscripts at

https://www.hindawi.com
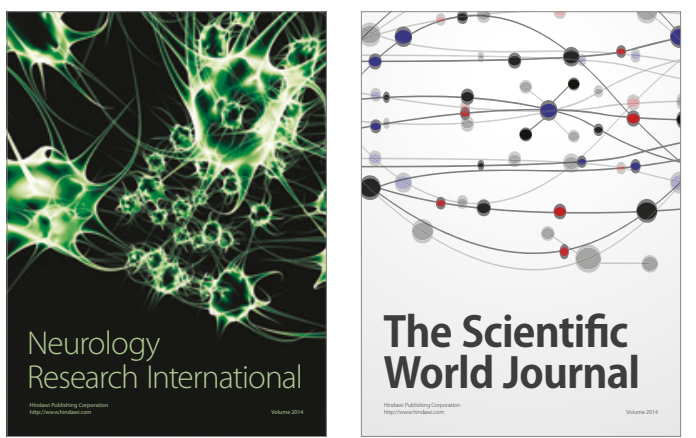

The Scientific World Journal

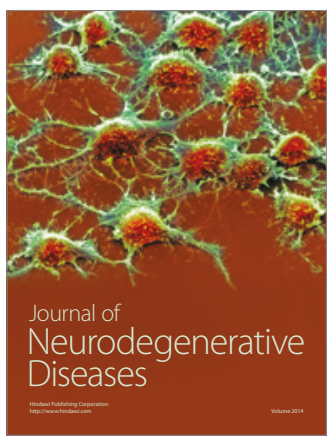

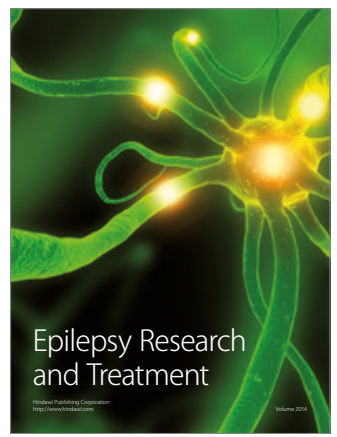

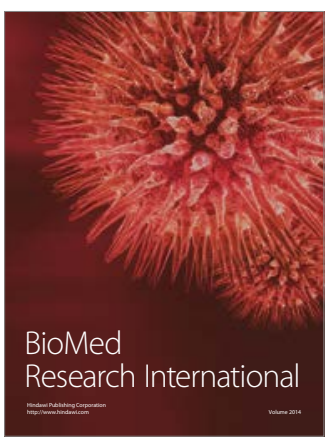

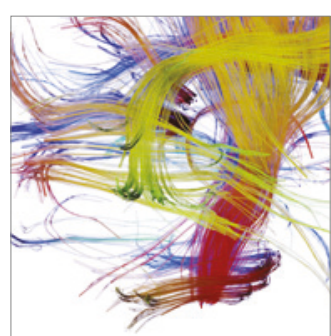

Brain Science

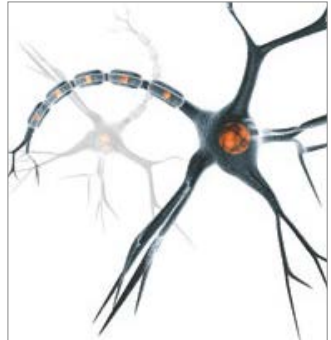

Neural Plasticity
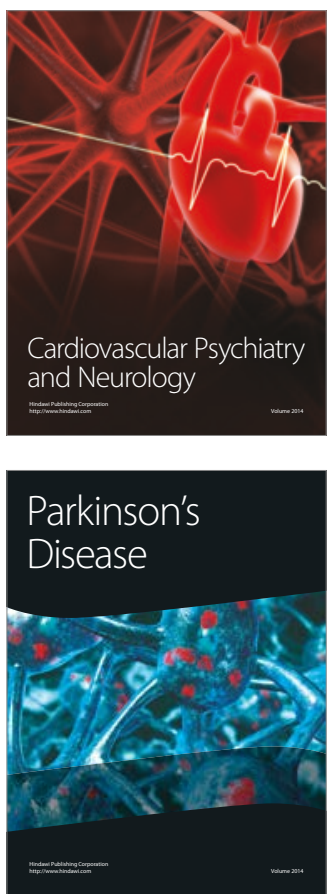